\title{
Quantum optics as enabling for quantum information science
}

\section{H. J. Kimble}

H. J. Kimble, "Quantum optics as enabling for quantum information science," Proc. SPIE 5842, Fluctuations and Noise in Photonics and Quantum Optics III, (23 May 2005); doi: 10.1117/12.609746

SPIE Event: SPIE Third International Symposium on Fluctuations and Noise, 2005, SPIE. Austin, Texas, United States 


\title{
Quantum Optics as Enabling for Quantum Information Science
}

\author{
H. J. Kimble \\ Norman Bridge Laboratory of Physics MC 12-33 \\ California Institute of Technology \\ Pasadena, CA 91125 USA
}

\begin{abstract}
A brief overview is given of the research activities in the Quantum Optics Group at Caltech related to the new science of Quantum Information.
\end{abstract}

Keywords: Quantum Optics, Cavity Quantum Electrodynamics, Quantum Information

\section{INTRODUCTION}

Across a broad front in physics, an exciting advance in recent years has been the increasing ability to observe and manipulate the dynamical processes of individual quantum systems. These advancing technical capabilities are being exploited for diverse investigations in Quantum Information Science related to radical new possibilities for the distribution and processing of information, as well as for precision measurement. A unifying theme is the attempt to exploit recently discovered possibilities in the microscopic realm of quantum mechanics to accomplish tasks that would otherwise be impossible by traditional classical means.

Within this setting, the Quantum Optics Group at Caltech has pursued several investigations related to strong coupling in optical physics whereby nonlinear interactions require only single atoms and photons. In qualitative terms, the research is directed towards advances beyond traditional nonlinear optics and laser physics into a new regime with dynamical processes involving atoms and photons taken one by one. We are striving to address fundamental issues related to quantum computation and communication, as for example in the realization of scalable quantum networks and photonic quantum computation, and to the general development of quantum information science and technology.

Brief descriptions of some of our recent work are given in the following sections, with reference to more detailed treatments in the scientific literature. With my apology, a comprehensive overview of the many exciting advances worldwide is beyond the scope of this abbreviated summary.

\section{CAVITY QUANTUM ELECTRODYNAMICS}

Over the past twenty years, an important physical system has been a single atom strongly coupled to the electromagnetic field of a high-Q (optical or microwave) cavity within the setting of cavity quantum electrodynamics (cavity QED). ${ }^{1}$ Diverse new phenomena in cavity QED include the realization of nonlinear interactions between single photons ${ }^{2}$ and the development of a laser in a regime of strong coupling that operates with "one-andthe-same-atom". ${ }^{3,4}$ Because of several unique advantages, cavity QED is playing an important role in the new science of quantum information, such as for the realization of complex quantum networks ${ }^{5}$ and for the investigation of quantum dynamics of single quantum systems. ${ }^{6}$

\subsection{A one-atom laser in the regime of strong coupling}

Although conventional lasers operate with a large number of intracavity atoms, the lasing properties of a single atom in a resonant cavity have been theoretically investigated extensively in the Quantum Optics community for more than a decade. We have utilized the capabilities developed in Ref. ${ }^{7}$ for the experimental realization of a oneatom laser operated in a regime of strong coupling. ${ }^{3,4}$ The observed characteristics of the atom-cavity system in this regime are qualitatively different from those of the familiar many atom case. Specifically, our measurements of intracavity photon number versus pump intensity exhibit "thresholdless" behavior, and we infer that the 

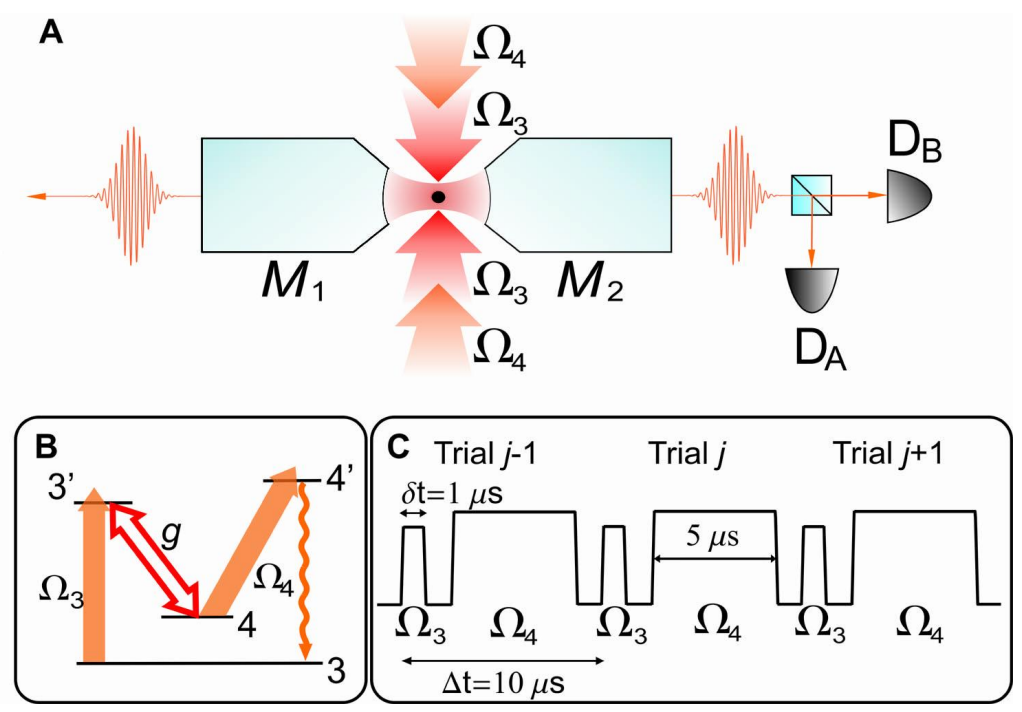

Figure 1. Illustration of the generation of single photons by one atom trapped in an optical cavity. (A) A single Cs atom is trapped in a cavity formed by the reflective surfaces of mirrors $\left(M_{1}, M_{2}\right)$ and is pumped by the external fields $\left(\Omega_{3}, \Omega_{4}\right) .^{7}$ (B) The relevant atomic levels of the Cs $D_{2}$ line at $852.4 \mathrm{~nm}$. Strong coupling at rate $g$ is achieved for the transition $F^{\prime}=3^{\prime} \rightarrow F=4$ near a cavity resonance, where $g=2 \pi \times 16 \mathrm{MHz}$. Atom and cavity decay rates $(\gamma, \kappa) / 2 \pi=(2.6 \mathrm{MHz}, 4.2 \mathrm{MHz})$. (C) The timing sequence for the generation of successive single-photons by way of the $\Omega_{3,4}$ fields.

output flux from the cavity mode exceeds that from atomic fluorescence by more than tenfold. Observations of the second-order intensity correlation function $g^{(2)}(\tau)$ demonstrate that our one-atom laser generates manifestly quantum (i.e., nonclassical) light that exhibits both photon antibunching $g^{(2)}(0)<g^{(2)}(\tau)$ and sub-Poissonian photon statistics $g^{(2)}(0)<1$. We have also carried out detailed comparisons between our measurements and theoretical models. ${ }^{4}$

\subsection{Generation of single photons "on demand" from one atom trapped in a cavity}

A crucial building-block for quantum information science is a deterministic source of single photons that generates one-quantum wavepackets in a well controlled spatiotemporal mode of the electromagnetic field. Moreover, the generation of single photons within the domain of strong coupling in cavity QED enables the reversible transfer of quantum states between atoms and photons, which is a fundamental primitive in protocols for the implementation of distributed quantum networks. ${ }^{8-10}$ A single-photon source consisting of a trapped atom strongly coupled to an optical cavity represents an ideal node for such a network, in which internal atomic states can be mapped to quantum states of the electromagnetic field by way of "dark" eigenstates of the atom-cavity system. Converting stationary qubits to flying qubits in this way enables distributed quantum entanglement over long distances by utilizing quantum repeaters.

As illustrated in Figure 1 and discussed in more detail in Ref., ${ }^{11}$ we have achieve the deterministic generation of single-photon pulses for a single atom strongly coupled to an optical cavity. The photon wavepackets are emitted as a Gaussian beam with temporal profile and repetition rate controlled by external driving fields. Referenced to the total cavity output, the generation efficiency for (un)polarized photons is (0.69 \pm 0.10$) 0.35 \pm$ 0.05. After correction for detector dark counts, the average suppression of two-photon to single-photon event probabilities is $R_{0}=20.8 \pm 1.8$, rising to $R_{0} \gtrsim 150$ at long times, where $R_{0}=1$ for a coherent state. In the absence of passive cavity losses, each generation attempt is inferred to succeed with probability $\phi_{G}=1.15 \pm 0.18$. These results represent a major step toward the realization of distributed quantum networks.

With this source for single photons, it now becomes feasible to implement a new scheme for scalable photonic quantum computation based on cavity assisted interaction between single-photon pulses. ${ }^{12}$ Here, the prototypical quantum controlled phase-flip gate between two single-photon pulses is achieved by successively reflecting 
the pulses from an optical cavity with a single-trapped atom. This protocol is robust to practical noise and experimental imperfections in current cavity-QED setups.

\subsection{Cavity QED "by the Numbers"}

We have developed a technique for the real-time determination of the number of atoms trapped within the mode of an optical cavity by monitoring the transmission of a weak probe beam. ${ }^{13}$ Continuous observation of atom number is accomplished in the strong coupling regime of cavity quantum electrodynamics and functions in concert with a cooling scheme for radial atomic motion. The probe transmission exhibits sudden steps from one plateau to the next in response to the time evolution of the intracavity atom number, from $N \geq 3$ to $N=2 \rightarrow 1 \rightarrow 0$ atoms. This is an important advance since many protocols in Quantum Information Science require multiple atoms to be trapped within the same cavity. Experimental efforts to combine ion trap technology with cavity QED are promising, but have not yet reached the regime of strong coupling. In Ref., ${ }^{13}$ the atom number is restricted to $N \lesssim 3$, but the novel detection scheme that we have developed may enable extensions to moderately larger atom numbers $N \lesssim 10$.

\subsection{Observation of the vacuum-Rabi splitting for an individual atom}

A cornerstone of optical physics is the interaction of a single atom with the electromagnetic field of a high quality resonator. Of particular importance is the regime of strong coupling, for which the frequency scale $g$ associated with reversible evolution for the atom-cavity system exceeds the rates $(\gamma, \kappa)$ for irreversible decay of atom and cavity field, respectively. In the domain of strong coupling, a photon emitted by the atom into the cavity mode is likely to be repeatedly absorbed and re-emitted at the single-quantum Rabi frequency $2 g$ before being irreversibly lost into the environment. This oscillatory exchange of excitation between atom and cavity field results from a normal-mode splitting in the eigenvalue spectrum of the atom-cavity system, and has been dubbed the vacuum-Rabi splitting.

Without exception experiments related to the vacuum-Rabi splitting in cavity QED with single atoms have required averaging over trials with many atoms $\left(\gtrsim 10^{3}\right)$ to obtain quantitative spectral information. By contrast, the implementation of complex algorithms in quantum information science requires the capability for repeated manipulation of an individual quantum system. With this goal in mind, we have succeeded in recording the entire vacuum-Rabi spectrum for one-and-the-same atom strongly coupled to the field of a high-finesse optical resonator. ${ }^{14}$ These measurements are made possible by a new Raman scheme for cooling atomic motion along the cavity axis for single atoms trapped within a state-insensitive intracavity FORT, ${ }^{7}$ with inferred atomic localization $\Delta z_{a x i a l} \simeq 33 \mathrm{~nm}$. Our measurements represent an important milestone towards the realization of more complex tasks in quantum computation and communication.

\subsection{Cavity QED with the whispering gallery modes of micro-toroidal resonators}

As illustrated in Fig. 2 and documented in Ref., ${ }^{15}$ toroidal microcavities offer exciting possibilities for achieving strong coupling for single atoms and photons. In collaboration with the group of Professor K. Vahala at Caltech, we are attempting to achieve strong coupling for one Cesium atom interacting with the external evanescent field of a toroidal microcavity. We have carried out numerical modeling of the optical modes that demonstrates a significant reduction of modal volume with respect to the whispering gallery modes of dielectric spheres, while retaining the high quality factors representative of spherical cavities. ${ }^{15}$ Moreover, numerical results for atomcavity coupling strength, critical atom number $N_{0}$, and critical photon number $n_{0}$ for one Cesium atom indicate substantial advantages relative to Fabry-Perot cavities. Modeling predicts coupling rates $g / 2 \pi$ exceeding $700 \mathrm{MHz}$ and critical atom numbers approaching $10^{-7}$ in optimized structures. Furthermore, preliminary experimental measurements of toroidal cavities at a wavelength of $852 \mathrm{~nm}$ indicate that quality factors in excess of 100 million can be obtained, which would result in strong coupling values of $\left(g /(2 \pi), n_{0}, N_{0}\right)=\left(86 \mathrm{MHz}, 4.6 \times 10^{-4}, 1.0 \times\right.$ $\left.10^{-3}\right)$. 


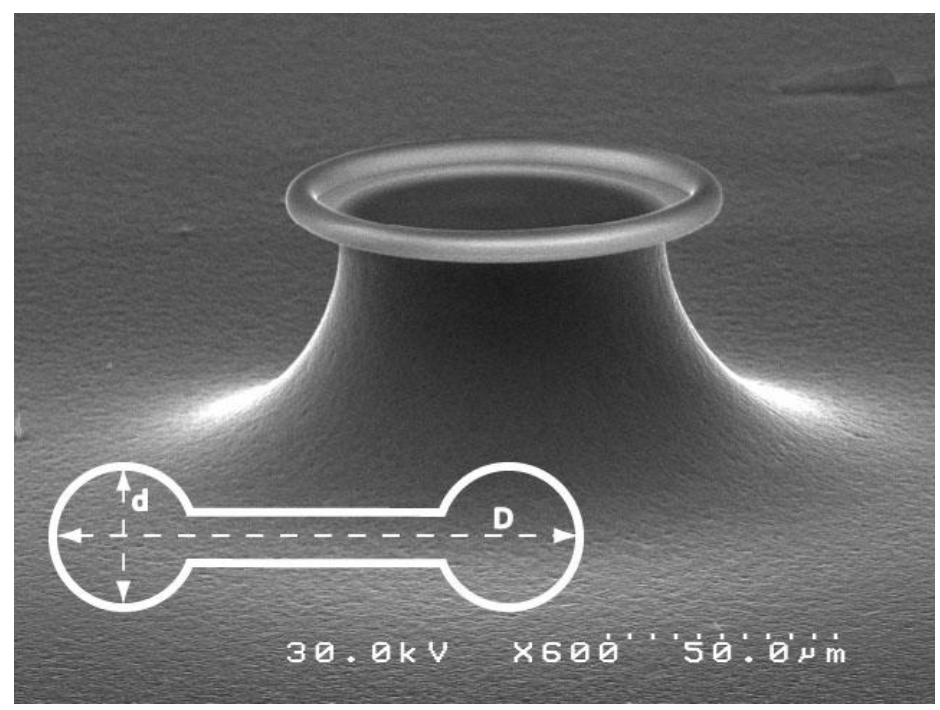

Figure 2. SEM micrograph of a toroidal microcavity. The principal and minor diameters are denoted by D and d, respectively.

\section{SCALABLE QUANTUM COMMUNICATION NETWORKS WITH ATOMIC ENSEMBLES}

There is an evolving understanding of the power of quantum measurement combined with conditional evolution for accomplishing diverse tasks in quantum information science. In this regard, a significant advance is the invention of a protocol by Duan, Lukin, Cirac, and Zoller (DLCZ) ${ }^{16}$ for the realization of scalable long-distance quantum communication and the distribution of entanglement over quantum networks. The theoretical proposal of $D L C Z^{16}$ is a probabilistic scheme based upon the entanglement of atomic ensembles via detection events of single photons in which the sources are intrinsically indistinguishable, and generates entanglement over long distances via a quantum repeater architecture. ${ }^{8}$ The $D L C Z$ scheme, with built-in quantum memory and entanglement purification, is well within the reach of current experiments and accomplishes the same objectives as previous more complex protocols that require still unattainable capabilities.

With the goal of implementing scalable quantum communication networks by way of the $D L C Z$ protocol, we have investigated manifestly quantum (i.e., nonclassical) correlations for photon pairs generated in the collective emission from an atomic ensemble. ${ }^{17}$ Our work includes the first observation of nonclassical photon correlations suitable for the implementation of the $D L C Z$ protocol for scalable quantum networks. ${ }^{18}$ As illustrated in Figure 3 , we have utilized the high degree of nonclassical correlation between photon pairs for the conditional generation of single photons from excitation stored within an ensemble of cold Cesium atoms. ${ }^{19}$ Conditioned upon an initial detection from field 1 at $852 \mathrm{~nm}$, a photon in field 2 at $894 \mathrm{~nm}$ is produced in a controlled fashion from excitation stored within the atomic ensemble. The single-quantum character of field 2 is demonstrated by the violation of a Cauchy-Schwarz inequality, namely $w\left(1_{2}, 1_{2} \mid 1_{1}\right)=0.24 \pm 0.05 \nsupseteq 1$, where $w\left(1_{2}, 1_{2} \mid 1_{1}\right)$ describes detection of two events $\left(1_{2}, 1_{2}\right)$ conditioned upon an initial detection event $1_{1}$.

Beyond these measurements, we have also explored the time dependence of nonclassical correlations for the $(1,2)$ fields generated by the atomic ensemble. ${ }^{20}$ The correlation function $R\left(t_{1}, t_{2}\right)$ for the ratio of cross to auto-correlations for the $(1,2)$ fields at times $\left(t_{1}, t_{2}\right)$ is found to have a maximum value $R^{\max }=292 \pm 57$, which significantly violates the Cauchy-Schwarz inequality $R \leq 1$ for classical fields. Decoherence of quantum correlations is observed over $\tau_{d} \simeq 175 \mathrm{~ns}$, and is described by a model that we have developed. A new scheme to mitigate the effects of decoherence is currently being implemented. The capabilities realized in our experiments provide important steps toward the implementation of the full protocol of $D L C Z$, which would enable the distribution and storage of entanglement among atomic ensembles distributed over a quantum network. 


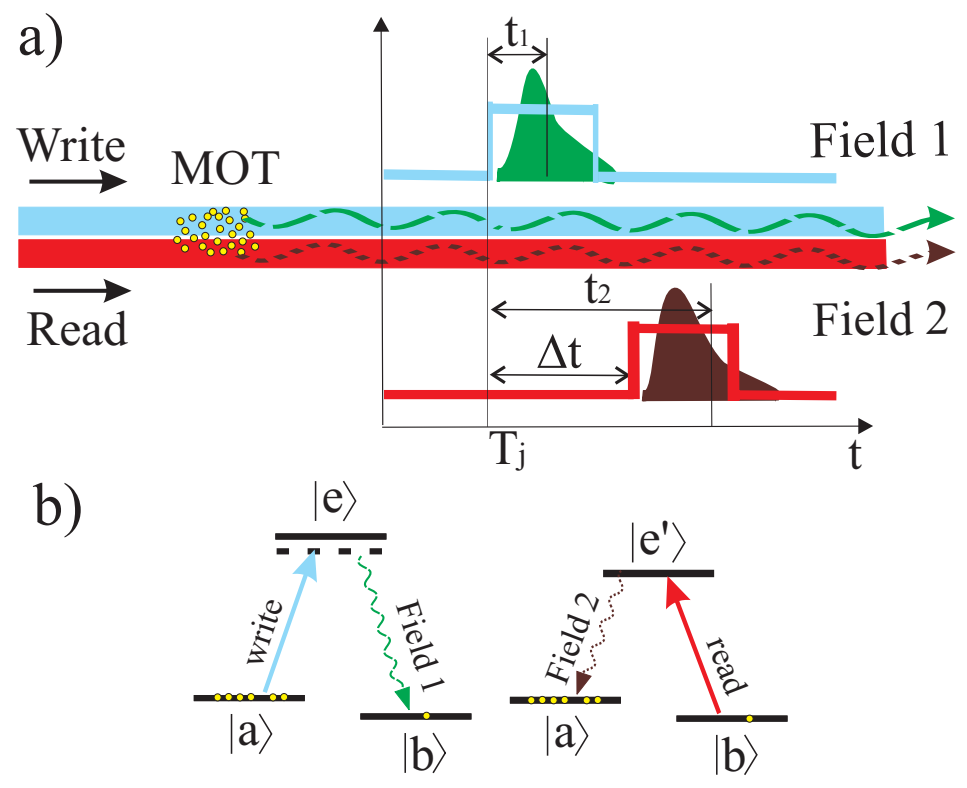

Figure 3. (a) Schematic of experiment for conditional generation of single photons. Write and read pulses sequentially propagate into a cloud of cold Cs atoms (MOT), generating the correlated output fields $(1,2)$. (b) The relevant atomic level scheme.

\section{ACKNOWLEDGMENTS}

I gratefully acknowledge the remarkable contributions of my many colleagues that have made this research possible. In particular, I thank the current members of the Caltech Quantum Optics Group, including Kevin Birnbaum, Andreea Boca, David Boozer, Warwick Bowen, Chin-Wen (James) Chou, Matthew Eichenfield, Daniel Felinto, Russell Miller, Tracy Northup, Sergey Polyakov, Hugues de Riedmatten, and Elizabeth Wilcut. Funding has been provided by the Caltech MURI Center for Quantum Networks, by the National Science Foundation (NSF), and by the Advanced Research and Development Activity (ARDA).

\section{REFERENCES}

1. For a review, see contributions in the Special Issue of Physica Scripta T76 (1998).

2. Q. A. Turchette, C. J. Hood, W. Lange, H. Mabuchi, and H. J. Kimble, Phys. Rev. Lett. 75, 4710 (1995).

3. J. McKeever, A. Boca, A. D. Boozer, J. R. Buck, and H. J. Kimble, Nature 425, 268 (2003).

4. A. D. Boozer, A. Boca, J. R. Buck, J. McKeever, and H. J. Kimble, Phys. Rev. A 70, 023814 (2003).

5. S. van Enk, J. I. Cirac, P. Zoller, H. J. Kimble, and H. Mabuchi, Fortschr. Phys. 46, 689 (1998).

6. C. J. Hood, T. W. Lynn, A. C. Doherty, A.S. Parkins, and H. J. Kimble, Science 287, 1447 (2000).

7. J. McKeever, J.R. Buck, A.D. Boozer, A. Kuzmich, H.-C.Nagerl, D.M. Stamper-Kurn, H.J. Kimble, Phys. Rev. Lett. 90, 133602 (2003).

8. H.-J. Briegel, S. van Enk, J.I. Cirac, P. Zoller, in The Physics of Quantum Information, D. Bouwmeester, A. Ekert, A. Zeilinger, Eds. (Springer, Berlin, 2000), pp. 192-197.

9. S. J. van Enk, J. I. Cirac, P. Zoller, H. Mabuchi, and H. J. Kimble, Journal of Modern Optics 44, 1727 (1997).

10. J. I. Cirac, S. J. van Enk, P. Zoller, H. J. Kimble, and H. Mabuchi, Physica Scripta T76, 223 (1998).

11. J. McKeever, A. Boca, A. D. Boozer, R. Miller, J. R. Buck, A. Kuzmich, and H. J. Kimble, Science 303, $1992(2004)$.

12. L.-M. Duan and H. J. Kimble, Phys. Rev. Lett. 92, 127902 (2004).

13. J. McKeever, J. R. Buck, A. D. Boozer, and H. J. Kimble, Phys. Rev. Lett. 92, 143601 (2004). 
14. A. Boca, R. Miller, K. M. Birnbaum, A. D. Boozer, J. McKeever, and H. J. Kimble, Phys. Rev. Lett. 93, 233603 (2004).

15. S. M. Spillane, T. J. Kippenberg, K. J. Vahala, K. W. Goh, E. Wilcut, and H. J. Kimble, Phys. Rev. A 71, 013817 (2005).

16. L.-M. Duan, M. Lukin, J. I. Cirac, and P. Zoller, Nature 414, 413 (2001).

17. For a review, see M. Lukin, Rev. Mod. Phys. 75, 457 (2003).

18. A. Kuzmich, W. P. Bowen, A. D. Boozer, A. Boca, C. W. Chou, L.-M. Duan, and H. J. Kimble, Nature 423, 731 (2003).

19. C. W. Chou, S. V. Polyakov, A. Kuzmich, and H. J. Kimble, Phys. Rev. Lett. 92, 213601 (2004).

20. S. V. Polyakov, C. W. Chou, D. Felinto and H. J. Kimble, Phys. Rev. Lett. 93, 263601 (2004). 